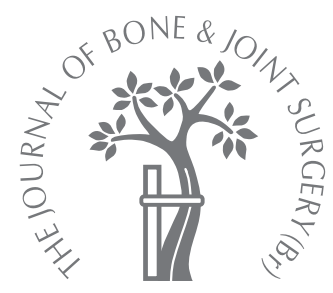

- CHILDREN'S ORTHOPAEDICS

\title{
Combined pelvic osteotomy for the bipartite acetabulum in late developmental dysplasia of the hip
}

\author{
A TEN-YEAR PROSPECTIVE STUDY
}

\author{
M. Rejholec \\ From the Al Razi \\ Orthopaedic \\ Hospital, Kuwait
}

M. Rejholec, $\mathrm{MD}, \mathrm{PhD}$, Consultant Orthopaedic Surgeon

Al Razi Orthopaedic Hospital, P. O. Box 4235 , Safat 13043 , Kuwait.

Correspondence should be sent to $\operatorname{Dr}$ M. Rejholec; e-mail: rejholec@hotmail.com

(C)2011 British Editorial Society of Bone and Joint Surgery doi:10.1302/0301-620X.93B2. $26147 \$ 2.00$

$J$ Bone Joint Surg $[\mathrm{Br}]$ 2011;93-B:257-61.

Received 9 June 2010

Accepted 27 October 2010

In late developmental dysplasia of the hip in childhood, the deformed dysplastic acetabulum is malaligned and has lost its shape due to pressure from the subluxed femoral head. The outer part of the acetabulum involves the upper part of the original acetabulum, thereby giving a bipartite appearance. A clear edge separates the outer from inner part which represents the lower part of the original acetabulum and has no direct contact with the femoral head.

Combined pelvic osteotomy (CPO) using a Lance acetabuloplasty with either a Salter or a Pemberton procedure restores the original shape and realigns the acetabulum. A total of 20 children ( 22 hips), with a mean age of 46 months (28 to 94 ) at primary operation underwent CPO with follow-up for between 12 and 132 months.

In each case concentric stable reduction with good acetabular cover was achieved and maintained throughout the period of follow-up.

In developmental dysplasia of the hip (DDH), subluxation of the femoral head, especially in older children, presents a difficult problem for those attempting surgical reconstruction of the acetabulum, ${ }^{1}$ which is not only dysplastic but also becomes deformed due to pressure from the subluxed femoral head on the upper part of the original acetabulum, resulting in a bipartite appearance. A clear edge separates the outer from the inner part which represents the lower part of the original acetabulum and has no direct contact with the femoral head. The outer part is lined by hyaline cartilage and its cranial margin is the acetabular labrum. It can therefore be easily distinguished from the false acetabulum. Conventional surgical techniques such as open reduction, femoral or pelvic osteotomy, or acetabuloplasty without reshaping the bipartite acetabulum, do not prevent the reduced head from slipping back into the outer part. Reshaping of the acetabulum alone without correcting the dysplasia of the original acetabulum can further aggravate damage to the growth area of the acetabular labrum by pressure from the subluxed head. ${ }^{1}$

In order to address these problems, a new type of combined pelvic osteotomy (CPO) was developed in which the Lance acetabuloplasty ${ }^{2}$ was combined with a Salter procedure. ${ }^{3,4}$ The outcome of this operation in 12 children with a minimum follow-up of five years has already been reported. ${ }^{1}$

As experience was gained over six years, it became apparent that this type of CPO was not suitable for all cases and a second type was developed. This combined the Lance acetabuloplasty with the Pemberton procedure. ${ }^{3,4}$ In this paper the mid-term results of both types of CPO are presented.

\section{Patients and Methods}

Between 1999 and 2009, 20 children underwent CPO. There were 15 girls and five boys. A total of 18 children were affected unilaterally, resulting in 22 hips studied.

The Lance acetabuloplasty and Salter osteotomy (Fig. 1) were used in 13 children (15 hips). Since 2005, the combination of Lance and Pemberton acetabuloplasties (Fig. 2) has been used in seven children (four girls, all unilateral).

For the first procedure the mean age at operation was 44 months (28 to 60) and the mean follow-up was 112 months (24 to 132 ). Of these 13 children, 12 were described in the previous study ${ }^{1}$ and their follow-up has been extended to between nine and 11 years. For the second procedure, the mean age at operation was 50 months (30 to 94 ) and the mean follow-up was 41 months (12 to 62). 


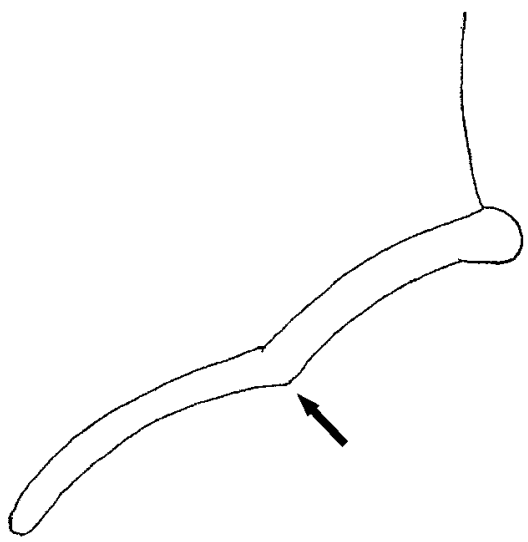

Fig. 1a

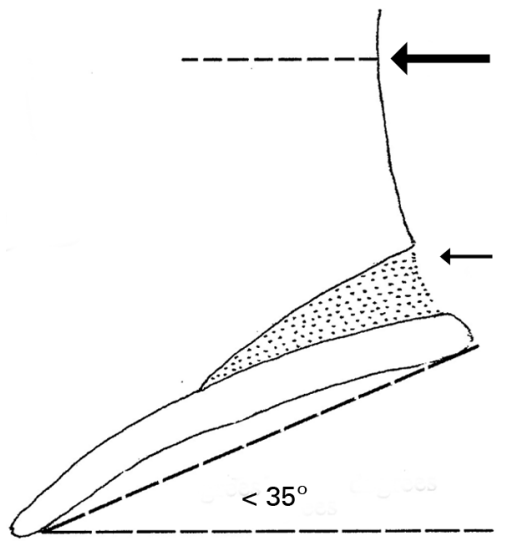

Fig. 1b

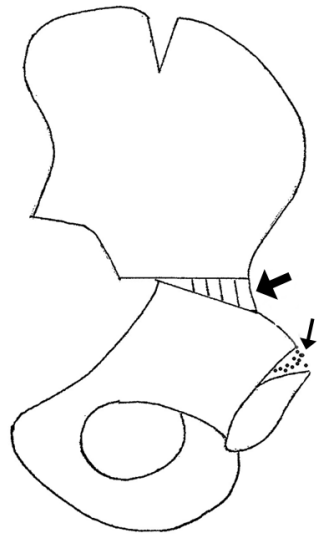

Fig. 1c

Diagrams of the Lance acetabuloplasty combined with the Salter osteotomy, showing a) the bipartite acetabulum displaying the border-like edge (arrow) between the inner and outer parts, b) the Lance acetabuloplasty reshaping the acetabulum (thin arrow) with persistent dysplasia $\left(\mathrm{AC}\right.$ angle $<35^{\circ}$ ) and the planned Salter osteotomy (thick arrow), and c) the hip after Lance acetabuloplasty with graft (thin arrow) and the modified Salter osteotomy with smaller bone graft (thick arrow).

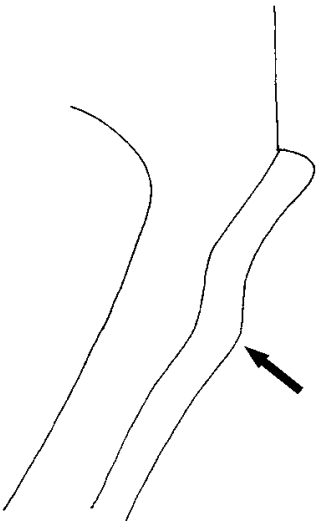

Fig. 2a

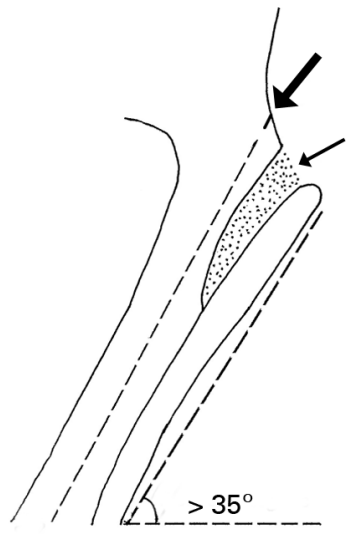

Fig. $2 b$

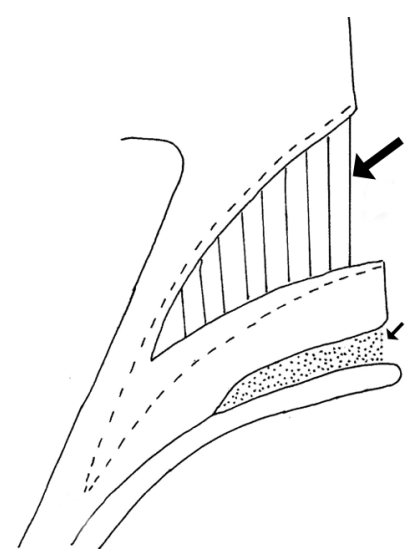

Fig. 2c

Diagrams of the Lance acetabuloplasty combined with the Pemberton osteotomy, showing a) the bipartite acetabulum with the border-like edge (arrow) between the inner and outer parts, b) the Lance acetabuloplasty reshaping the acetabulum (thin arrow) with persistent severe dysplasia (AC angle $>35^{\circ}$ ) and the planned Pemberton procedure (thick arrow), and c) the hip after Lance acetabuloplasty with inserted bone graft (thin arrow) and the Pemberton osteotomy with graft (thick arrow).

In older children (Figs 3 to 5) the bipartite acetabulum can be seen on plain radiographs, but in those under two years of age it may only be visible on arthrography. In these children the deformity is still reversible and may be treated successfully by conventional means. ${ }^{1}$ In older children, the bipartite acetabulum is also visible during surgery. The outer part, with its white bright hyaline cartilage, may be mistaken for the true acetabulum and the clear border-like edge, which separates this outer from the inner part, may be considered as the incisura acetabuli. The proximal border of the true acetabulum is demarcated by the reflected head of the rectus femoris and by the labrum, which is very often flattened and deformed by pressure of the subluxed head (Fig. 6).

Operative technique. This has been previously described, ${ }^{1}$ but the details are important in order to select the correct type of CPO. After a routine anterior approach, the joint capsule should be exposed and cleaned in order to define the border between the capsule and the iliac bone. The reflected head of rectus femoris is a guide to define the proximal border of the true acetabulum. A T-shaped capsulotomy is performed, with the horizontal component parallel to the acetabular margin, leaving about $5 \mathrm{~mm}$ of capsule on the acetabular rim for capsulorrhaphy; the vertical component follows the lateral border of the femoral neck to obtain a proper view of the acetabulum. It is important to make the medial flap of the T-incision big enough to cover the reduced femoral head completely from its anterior side during capsulorrhaphy. When the joint is properly exposed, the ligamentum teres, the transverse ligament and the pulvinar acetabuli are excised and the joint cartilage is cleansed with a gauze swab. Special care should be taken to define the border-like edge that separates the inner and outer parts of the acetabulum. As noted above, it is important not to misinterpret this border as the incisura acetabuli (Fig. 6). 


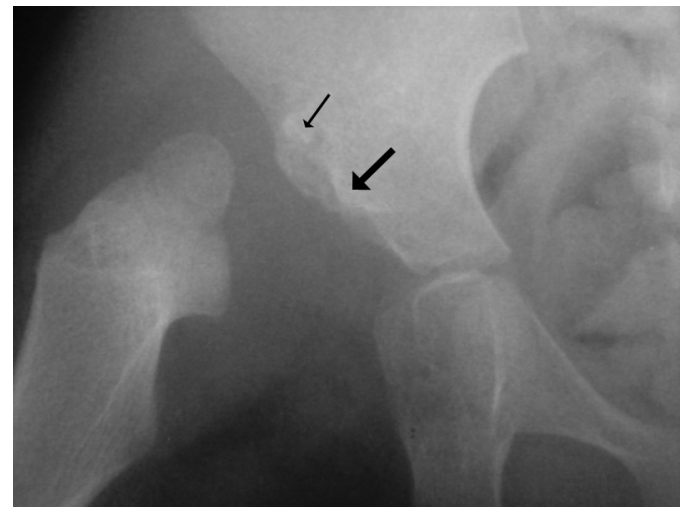

Fig. 3a

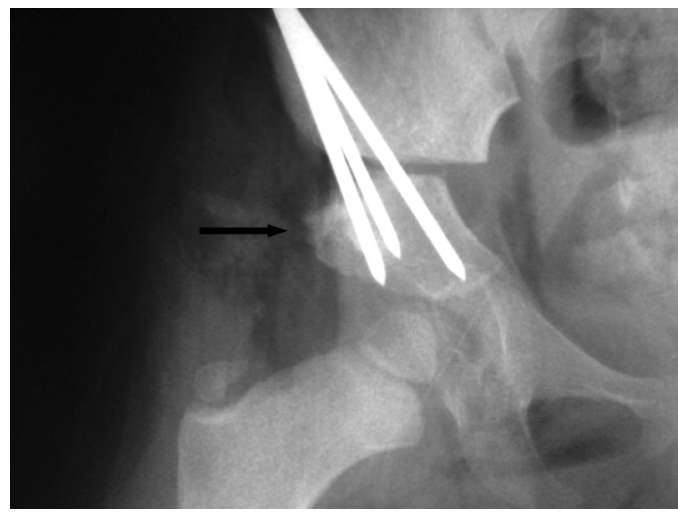

Fig. 3b

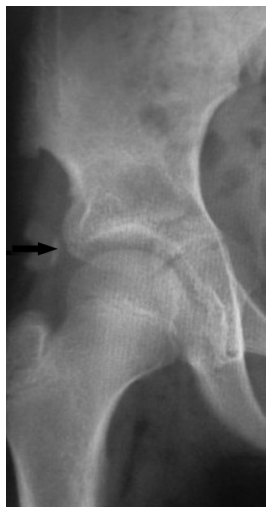

Fig. 3c

Radiographs of a 35-month-old child with subluxation of the femoral head, showing a) pre-operatively the border-like edge of the bipartite acetabulum (thick arrow) and the proximal margin of the true acetabulum (thin arrow), b) the Lance acetabuloplasty and modified Salter innominate osteotomy, with the bone graft (arrow) between the subchondral bone and the cartilage of the outer part of the acetabulum, and c) concentric reduction with good cover at ten years post-operatively. The incorporated bone graft is still visible (arrow).

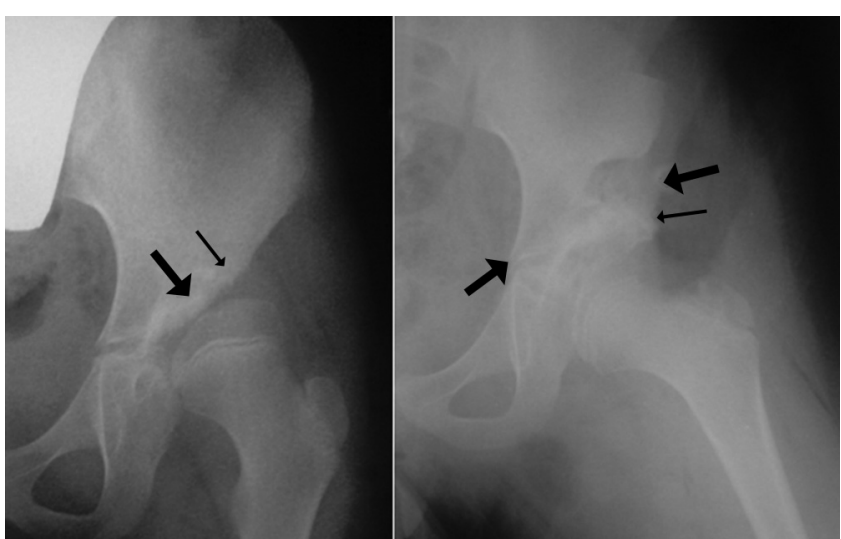

Fig. 4

Radiographs of the hip of a girl aged seven years and ten months, showing on the left a bipartite deformed acetabulum with severe dysplasia (AC angle of $45^{\circ}$ ) and subluxation of the femoral head (thick arrow, border-like edge of the bipartite acetabulum; thin arrow, proximal margin of the true acetabulum), and on the right a Lance acetabuloplasty (thin arrow) and Pemberton osteotomy (thick arrows) with full correction of both deformity and dysplasia.

The ligamentum teres, if preserved, can be used as a guide. The border-like edge can be seen in Figures 1 to 6 .

Proper exposure of the joint cavity is essential for further decision making. The outer part of the acetabulum is defined and a Lance acetabuloplasty ${ }^{2}$ is performed as described in the previous study. ${ }^{1}$ This restores the spherical shape of the acetabulum and eliminates the border-like edge.

The still malaligned acetabulum is assessed to determine the second procedure. A stability test is carried out to see how much abduction and flexion are needed to achieve a stable hip. If the hip is stable in about $30^{\circ}$ of abduction and flexion, a Salter procedure is indicated. If more flexion and abduction are needed, the dysplasia is advanced and a
Pemberton acetabuloplasty is more appropriate. In unclear cases, a peri-operative radiograph should be taken to measure the acetabular cover (AC) angle. If this is $>35^{\circ}$, the Pemberton procedure will give better cover as it has a greater capacity for correction of the AC angle. ${ }^{1,3-6}$ It would, however, be a serious technical mistake to use the Pemberton osteotomy for all types of dysplasia. In mild cases the procedure, with its capacity to correct greater deformities, may partially or completely close entry to the acetabulum, thereby making concentric reduction difficult or even impossible.

Figures 1 and 2 show the appropriate indications for the use of the Salter and Pemberton procedures. Figures 3 and 4 show the pre- and post-operative radiographs of these procedures.

The Salter osteotomy is modified, with a shift of the distal fragment anterolaterally and the use of smaller bone graft to give a greater correction and prevent the unwelcome long-leg effect of the classic Salter technique. ${ }^{1,4,6}$

A tight capsulorrhaphy with the hip in $30^{\circ}$ of abduction, $20^{\circ}$ of flexion and $15^{\circ}$ of internal rotation is important to prevent any lateralisation, and a plaster spica cast is applied in the same position. The cast is removed at six weeks and the child is allowed to mobilise with the aid of hydrotherapy. During the first five years of the study the cast was replaced at six weeks by broomstick plasters for a further four weeks. However, we subsequently stopped using broomstick plasters under these circumstances.

\section{Results}

In each case, concentric reduction with sufficient cover was achieved (Figs 3c and 4). All hips were graded as excellent according to the McKay classification. ${ }^{3}$ This indicates a stable, painless hip with no limp, a negative Trendelenburg sign and a full range of movement. All but one was graded radiologically as excellent (Severin I). ${ }^{1,4}$ This indicates a normally-shaped femoral head and a centre-edge angle 


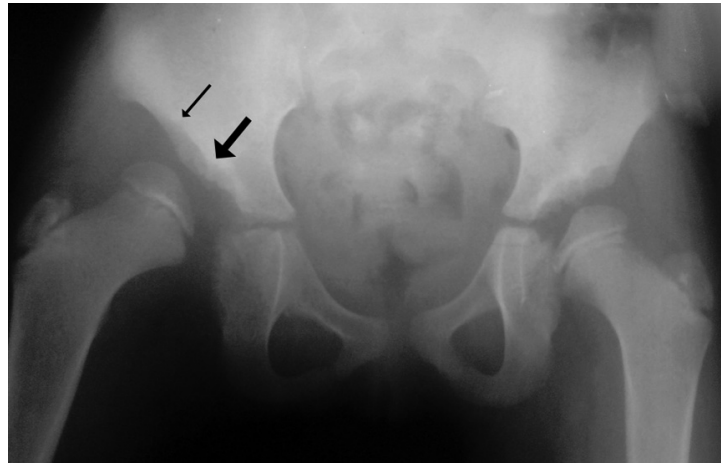

Fig. 5a

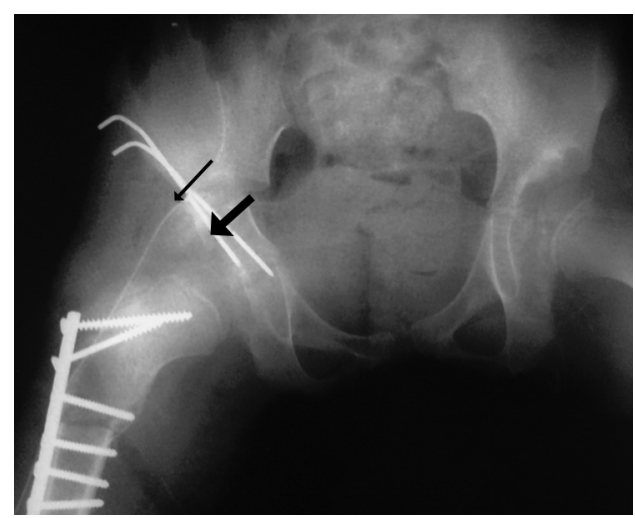

Fig. $5 b$

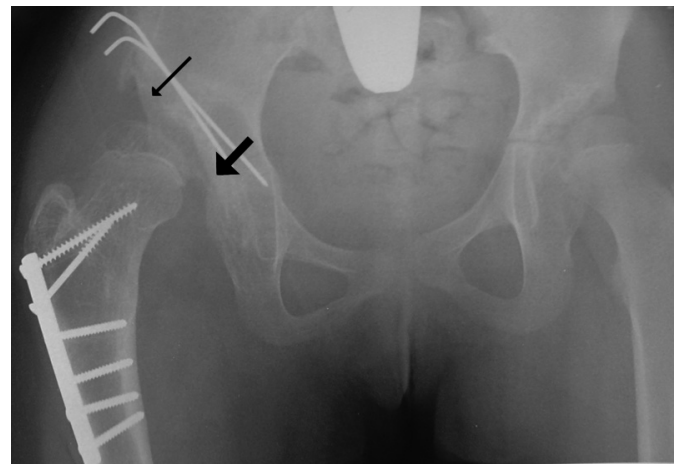

Fig. 5c

Radiographs of the hip of a seven-year-old girl with subluxation of the right hip (thick arrow, border-like edge of the bipartite acetabulum; thin arrow, proximal margin of the true acetabulum) showing a) the pre-operative appearance, b) after open reduction and Salter and femoral osteotomies without reshaping of the acetabulum, with reduction of the femoral head, and c) at two years post-operatively the femoral head has slipped into the outer part of the uncorrected bipartite acetabulum.

$>20^{\circ}$. One case was graded Severin II, which is defined as a slight deformity of the femoral head with concentric reduction and a centre-edge angle $>20^{\circ}$. Even in cases such as this, where possible delayed growth of the acetabular rim can occur because of the concomitant innominate osteotomy, the cover of the femoral head was sufficient, with a centre-edge angle of $25^{\circ}$ at eight years after operation. ${ }^{4}$ The

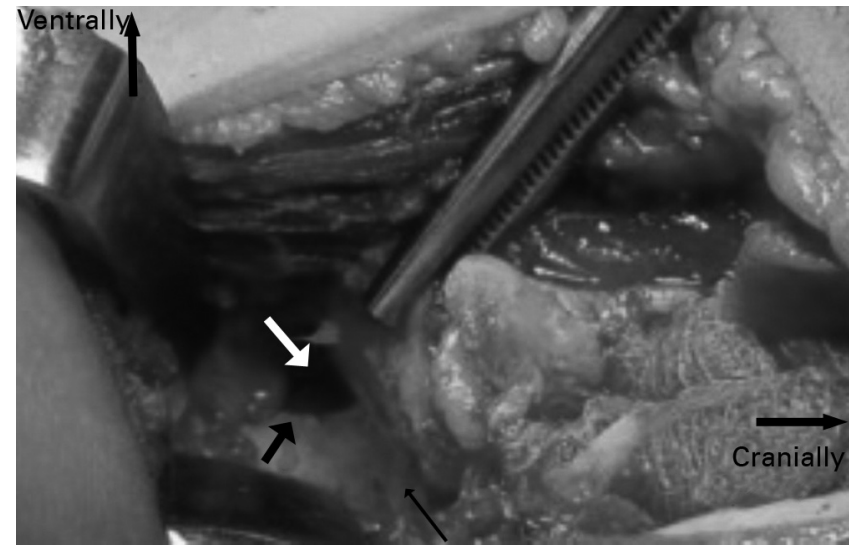

Fig. 6

Peri-operative photograph showing the bipartite acetabulum of a 43month-old child. The thick black arrow shows the border-like edge between the inner and outer parts of the acetabulum. The thin black arrow shows the proximal border of the acetabulum. The white arrow shows the inner part of the acetabulum. The orientation of the acetabulum is marked by arrows showing cranial and ventral directions.

mild deformity of the femoral head may have been due to ischaemia. There was no case of ischaemic necrosis on the acetabular side.

\section{Discussion}

The criteria for the use of the Salter and Pemberton osteotomies $^{3-6}$ as well as the risk of ischaemic necrosis and early arthritis ${ }^{7,8}$ were discussed in a previous study. ${ }^{1}$

Residual subluxation requiring repeated surgery makes the risk of these complications very high.

A typical case of redislocation is shown in Figure 5. This child was not included in the series and was referred by another surgeon. Technically correct conventional surgery had failed to restore a spherical acetabulum and within two years there was subluxation to the outer part of the acetabulum. The residual subluxation will need further surgery to stabilise the hip, and the long-term prognosis remains doubtful.

In 1998 Fixsen and $\mathrm{Li}^{9}$ published their study on the treatment of subluxation of the hip in children over the age of four years. Their work, together with the author's personal experience with similar cases, became the main inspiration for the introduction of CPO in $1999 .{ }^{1}$ Fixsen and $\mathrm{Li}^{9}$ presented ten children with different methods of conventional surgical treatment, such as open reduction, femoral osteotomy, shelf arthroplasty and Salter and Pemberton osteotomy, with a follow-up of seven to ten years. In four cases one revision was necessary, and in another two revisions were required. Notwithstanding these procedures there were three cases of residual subluxation of the hip and four of ischaemic necrosis of the femoral head. 
The indication for femoral osteotomy in children over the age of $\operatorname{six}^{10}$ was discussed in the first study. ${ }^{1}$

The Lance osteotomy alone was evaluated by Weber et al. ${ }^{11}$ In 58 patients ( 78 hips) with a follow-up of between three and 11 years there was a progressive increase in the incidence of residual dysplasia from $35 \%$ to $51 \%$.

One of the reasons to re-evaluate the earlier group of $\mathrm{CPO}^{1}$ was to look for late dysplasia. None was found.

Tönnis and Sprafke ${ }^{12}$ published the results of a modified Lance acetabuloplasty combined with femoral varus derotation osteotomy in 254 cases, with a follow-up between three and seven years. Patients were divided according to their age at operation. In patients between one and six years old, $20 \%$ had residual acetabular dysplasia, and in patients between six and ten years this had risen to $40 \%$.

The Lance osteotomy has been blamed for damage to the area of the acetabulum responsible for its growth, ${ }^{11}$ and this is an argument against CPO. However, this concern can be addressed in two ways. First, the area is probably already damaged by pressure from the subluxed head, and delayed growth is compensated for by the additional osteotomy. Secondly, the follow-up over ten years without any case of subluxation or dysplasia suggests that the possible delay in acetabular growth was compensated for by the additional osteotomy.

Only plain radiographs were taken in this study. No special investigations of the blood supply to the femoral head and acetabular rim, such as an MRI, isotope bone scan or arteriography, were performed. This might have required general anaesthesia for the affected children and caused an unnecessary financial burden for the parents and the healthcare provider.

To date, and with practically the same follow-up, the problems encountered by Fixsen and $\mathrm{Li}^{9}{ }^{9}$ Weber et $\mathrm{al}^{11}$ and Tönnis and Sprafke ${ }^{12}$ have not been observed after CPO.

\section{Supplementary material}

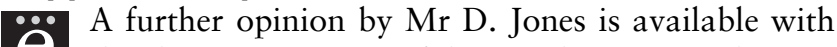
C the electronic version of this article on our website at www.jbjs.org.uk/education/further-opinions

\section{Listen live}

Listen to the abstract of this article at www.jbjs.org.uk/interactive/audio

No benefits in any form have been received or will be received from a commercial party related directly or indirectly to the subject of this article.

\section{References}

1. Rejholec M. Combined pelvic osteotomy for deformed dysplastic acetabula: a 5-year prospective study. J Orthop Surg 2007;15:347-51.

2. Lance PM. Constitution d'une butee ostéoplastique dans les luxations et sub-luxations congénitales de la hanche. Presse Med 1925:33:945-8 (in French).

3. McKay DW. A comparison of the innominate and the pericapsular osteotomy in the treatment of congenital dislocation of the hip. Clin Orthop 1974;98:124-32.

4. Rejholec M. Osteotomy of the pelvis. Acta Universitatis Carolinae Medica Monographia CXL III 1993;1-140.

5. Rejholec M. Preliminary report: Pemberton acetabuloplasty in the treatment of severe acetabular dysplasia. Kuwait Medical Journal 2000;32:44-6

6. Salter RB, Dubos JP. The first fifteen year's personal experience with innominate osteotomy in the treatment of congenital dislocation and subluxation of the hip. Clin Orthop 1974;98:72-103

7. Kershaw CJ, Ware HE, Pattinson R, Fixsen JA. Revision of failed open reduction of congenital dislocation of the hip. J Bone Joint Surg [Br] 1993;75-B:744-9.

8. Gulman B, Tuncay IC, Dabak W, Karaismailoglu N. Salter's innominate osteotomy in the treatment of congenital hip dislocation: a long-term review. J Pediatr Orthop 1994;14:662-6.

9. Fixsen JA, Li PL. The treatment of subluxation of the hip in children over the age of four years. J Bone Joint Surg [Br] 1998;80-B:757-61.

10. Rejholec M, Stryhal F. Behavior of the proximal femur during the treatment of congenital dysplasia of the hip: a clinical long-term study. J Pediatr Orthop 1991;11:506-13

11. Weber M, Wirtz D, Jaeschke C, Niethard FU. Growth disorders of the acetabular roof after acetabuloplasty in congenital hip dysplasia. Z Orthop Ihre Grenzgeb 1998;136:525-34 (in German).

12. Tönnis D, Sprafke K. New results of a modified acetabuloplasty after Lance combined with detorsion-varus-osteotomy. Z Orthop Ihre Grenzgeb 1977;115:743-52 (in German). 